PAPER I

\title{
Probabilistic simulation of fire scenarios
}

In: Nuclear Engineering and Design 2003.

Vol. 224, No. 3, pp. 301-311.

Copyright Elsevier 2003.

Reprinted with permission from the publisher. 


\title{
Probabilistic simulation of fire scenarios
}

\author{
Simo Hostikka*, Olavi Keski-Rahkonen \\ VTT Building and Transport, PO Box 1803, FIN-02044 VTT, Finland
}

Received 15 November 2002; received in revised form 26 March 2003; accepted 29 March 2003

\begin{abstract}
A risk analysis tool is developed for computation of the distributions of fire model output variables. The tool, called Probabilistic Fire Simulator (PFS), combines Monte Carlo simulation and CFAST, a two-zone fire model. In this work, the tool is used to estimate the failure probability of redundant cables in a cable tunnel fire, and the failure and smoke filling probabilities in an electronics room during an electronics cabinet fire. Sensitivity of the output variables to the input variables is calculated in terms of the rank order correlations. The use of the rank order correlations allows the user to identify both modelling parameters and actual facility properties that have the most influence on the results. Various steps of the simulation process, i.e. data collection, generation of the input distributions, modelling assumptions, definition of the output variables and the actual simulation, are described.

(C) 2003 Elsevier Science B.V. All rights reserved.
\end{abstract}

\section{Introduction}

\subsection{Setting the problem}

Traditionally, the deterministic fire models have been used to estimate the consequences of the fire with some given set of input variables. Presuming the model is principally valid for the given problem, the uncertainty of the prediction depends on, how the uncertainties in input values are transferred through the system described by the model. As the systems are usually non-linear and rather complicated, the classical analytic methods of estimation of random error propagation are of little use. The possible uncertainty or distribution of the input variables can be taken into account by manually varying the inputs within allowable and rather narrow limits. This is mathematically a fairly well-posed problem, and a rough range of error

\footnotetext{
* Corresponding author. Tel.: +358-9-456-4839; fax: +358-9-456-4815.

E-mail address: simo.bostikka@vtt.fi (S. Hostikka).
}

can be established for the target function by carrying out a fairly small number of such calculations.

If more complete information of the error distribution is needed, Monte Carlo techniques should be used. In Monte Carlo, a large number of samples is randomly chosen from the input space and mapped through the system into the target distribution. In demanding error analyses this has been a standard technique for some time.

The problem is somewhat different when fire models are used for estimation of target fire safety. To enlighten the difference, we consider two examples. In Example 1, the scenario is taken as the author's office. While writing this paper, the computer might catch fire. The target question is: what is the probability that the fire spreads outside the room of origin, i.e. outside the author's office?

While this target question might be interesting individually, a more relevant question for the fire safety of our office building would be (Example 2): given a fire breaks out in an office, what is the probability it spreads outside the room of origin? Since various 


\begin{tabular}{|c|c|}
\hline \multicolumn{2}{|c|}{ Nomenclature } \\
\hline$A_{\text {fuel }}$ & $\begin{array}{l}\text { area of the fuel surface inside the } \\
\text { electronics cabinet }\end{array}$ \\
\hline$A_{\mathrm{e}}$ & area of the cabinet exhaust opening \\
\hline$A_{\mathrm{i}}$ & area of the cabinet inflow opening \\
\hline$F_{i}$ & $\begin{array}{l}\text { probability distribution function } \\
\text { of variable } i\end{array}$ \\
\hline$f_{i}$ & $\begin{array}{l}\text { probability density function of } \\
\text { variable } i\end{array}$ \\
\hline$g$ & limit state function \\
\hline$H_{\mathrm{v}}$ & height between the cabinet openings \\
\hline$n$ & number of random variables \\
\hline$P_{\mathrm{A}}$ & probability of event A \\
\hline$P_{\text {ign }}$ & probability of ignition \\
\hline$\dot{Q}$ & rate of heat release (RHR) \\
\hline$\dot{Q}_{\text {fuel }}^{\prime \prime}$ & rate of heat release per fuel area \\
\hline$X$ & vector of random variables \\
\hline$x$ & random variable \\
\hline$t$ & time \\
\hline$t_{\mathrm{g}}$ & RHR growth time \\
\hline$t_{\mathrm{d}}$ & starting time of the RHR decay phase \\
\hline$z$ & height \\
\hline$z_{\text {target }}$ & height of the target component \\
\hline$z_{\text {source }}$ & height of the fire source \\
\hline \multicolumn{2}{|c|}{ Greek letters } \\
\hline$\chi$ & mbustion efficiency \\
\hline$\phi_{x}$ & int probability density function \\
\hline$\tau$ & HR decay time \\
\hline
\end{tabular}

rooms do not differ considerably from each other, a deterministic room fire model could be used as well for the both cases. Since in Example 2, the room of ignition could be any of the rooms in the building, an averaging over the rooms is needed to answer the final question. For Example 1, we assess only one room, for Example 2, some 100 rooms need to be assessed. To find an answer to Example 2, we have to take averages over the whole set of these 100 rooms forming our office building.

\subsection{Goal of the paper}

The goal of this study is to develop a calculation tool for Example 2 in the form of a probabilistic fire simulation. A specific task is the prediction of the failure probabilities of specified items in fires. If the size of the set to be assessed grows, individual assessment of each scenario becomes uneconomic. Describing variables of the set to be assessed as distributions a risk analysis model can be developed using the Monte Carlo simulation. Formally, deterministic calculations are the same for Examples 1 and 2. Backed by some experience we believe, that input distributions are generally much narrower for Example 1 than for Example 2. Typically, for Example 1 , an input variable is normally distributed, whereas for Example 2, the same variable has lognormal or other skewed distribution. While using this approach, we presume further, that the epistemic uncertainty of the applied deterministic model is small as compared with the uncertainties caused by input distributions. When this is not the case, Monte Carlo is not the way to handle the problem. Instead, the modelling must be improved. We have already built this possibility in our tool: starting economically from correlations for simple cases, we can change to a zone model, and ultimately go to computational fluid dynamics.

Commercial Monte Carlo simulation software @ RISK $^{1}$ is used for the random sampling and post processing. A two-zone model CFAST (Peacock et al., 1993) is used to model smoke spreading and gas temperature during the fire. The risk analysis software and CFAST are combined in a spreadsheet computing environment. The tool, called Probabilistic Fire Simulator (PFS), is intended to be fully general and applicable to any fire scenario amenable to deterministic numerical simulation. The main outcome of the new tool is the automatic generation of the distributions of the selected result variables, for example, component failure time. The sensitivity of the output variables to the input variables can be calculated in terms of the rank order correlations. The use of the rank order correlations allows the user to simultaneously identify both the modelling parameters and the actual facility properties that have the most influence on the results. Typically, the simulation process consists of the data collection, generation of the input distributions, modelling and assumptions, definition of the output variables and the actual Monte Carlo simulation.

The validity of the models used is not discussed here. We do not compare our results with experiments

\footnotetext{
$1 @$ RISK is a product of Palisade Corporation, Newfield, NY.
} 
either, because the results from some 1000 random experiments are not available for any real fire scenario. There is only one potential source on such data: fire statistics. Even the best statistical data would not contain information detailed enough for comparison with deterministic calculations. In the future, we are going to enlarge the input database, and improve the fire models for the calculation of true scenarios allowing comparison with fire statistics directly. So far it is too early to say whether this goal is realistic. Meanwhile, we concentrate on the collection of good statistical data for input distributions, and use deterministic fire models, which are well evaluated.

Two example scenarios are studied here: a fire in a nuclear power plant cable tunnel and a fire in an electronics room. The cable tunnel fire was studied experimentally by Mangs and Keski-Rahkonen (1997) and theoretically by Keski-Rahkonen and Hostikka (1999). These studies showed that the CFAST two-zone model can be used to predict the thermal environment of a cable tunnel fire, at least in its early stages. Here, the effects of the input variables, like tunnel geometry and fire source properties, are studied by choosing them randomly. The input distributions are based on the statistics collected from the power plant.

The electronics room fire was previously studied by Eerikäinen and Huhtanen (1991). They used computational fluid dynamics to predict the thermal environment inside the room, when one electronics cabinet is burning. The same scenario is studied here using the two-zone model. The fire source is selected randomly based on the collected distribution of different cabinet types.

\section{Monte Carlo simulation}

The question set by the probabilistic safety assessment process is usually: "What is the probability that a certain component or system is lost during a fire?" This probability is a function of all possible factors that may affect on the development of the fire and the systems reaction to it. This question has not been dealt with exactly for fire in this form, but similar systems in other fields have been studied extensively (Spiegel, 1980; Vose, 1996). Here we adapt this general theory for our specific fire problem.
Let us denote the group of affecting variables by a vector $\boldsymbol{X}=\left(X_{1}, X_{2}, \ldots, X_{n}\right)^{\mathrm{T}}$ and the corresponding density functions by $f_{i}$ and distribution functions by $F_{i}$. The occurrence of the target event $\mathrm{A}$ is indicated by a limit state function $g(t, \boldsymbol{x})$, which depends on time $t$ and vector $x$ containing the values of the random variables. As an example of the target event, we consider the loss of some component. The limit state condition is now defined using function $g(t, \boldsymbol{x})$ :

$g(t, \boldsymbol{x}) \leq 0$, if the component is lost at time $t$ $g(t, \boldsymbol{x})>0$, if the component is not lost at time $t$

Other possible target events are, for example, heat detector activation and smoke filling. The development of fire and the response of the components under consideration are assumed to be fully deterministic processes where the same initial and boundary conditions always lead to the same final state. With this assumption, the probability of event A can now be calculated by the integral

$P_{\mathrm{A}}(t)=\iint_{\{\boldsymbol{x} \mid g(t, \boldsymbol{x}) \leq 0\}} \cdots \int \phi_{x}(\boldsymbol{x}) \mathrm{d} x_{i}$

where $\phi_{x}$ is the joint density function of variables $\boldsymbol{X}$. Generally, variables $\boldsymbol{X}$ are dependent, and $\phi_{x}(\boldsymbol{x}) \neq$ $\prod_{i=1}^{n} f_{i}\left(x_{i}\right)$.

In this work, the probability $P_{\mathrm{A}}$ is calculated using Monte Carlo simulations where input variables are sampled randomly from the distributions $F_{i}$. If $g(t, \boldsymbol{x})$ is expensive to evaluate, a stratified sampling technique should be used. In Latin Hypercube sampling (LHS) the $n$-dimensional parameter space is divided into $N^{n}$ cells (McKay et al., 1979). Each random variable is sampled in fully stratified way and then these samples are attached randomly to produce $N$ samples from $n$ dimensional space. The advantage of this approach is that the random samples are generated from all the ranges of possible values, thus giving insight into the tails of the probability distributions. A procedure for obtaining a Latin Hypercube sample for multiple, spatially correlated variables is given by Stein (1987). He showed that LHS will decrease the variance of the resulting integral relative to the simple random sampling whenever the sample size $N$ is larger than the number of variables $n$. However, the amount of reduction increases with the degree of additivity in the random quantities on which the function being simulated depends. In fire simulations, the simulation 
result may often be a strongly non-linear function of the input variables. For this reason, we cannot expect that LHS would drastically decrease the variances of the probability integrals. Problems related to LHS with small sample sizes are discussed by Hofer (1999) as well as by Pebesma and Heuvelink (1999).

The sensitivity of the output $y$ to the different input variables $x$ is studied by calculating the Spearman's rank-order correlation coefficients (RCC). A value's "rank" is determined by its position within the min-max range of possible values for the variable. $\mathrm{RCC}$ is then calculated as

$\mathrm{RCC}=1-\frac{6 \sum d^{2}}{m\left(m^{2}-1\right)}$

where $d$ is the difference between ranks of corresponding $x$ and $y$, and $m$ is the number of data pairs. RCC is independent of the distribution of the initial variable. The significance of the RCC values should be studied with the methods of statistical testing. In case of small datasets, the actual values of RCC should be interpreted with caution due to the possible spurious correlations inside the input data (Hofer, 1999).

\section{Fire modelling}

The transport of heat and smoke is simulated using a multi-room two-zone model CFAST (Peacock et al., 1993). It assumes two uniform layers, hot and cold, in each room of the building and solves the heat and mass balance equations for each room. A PFS worksheet is used to generate the input data for CFAST. The user may combine any experimental information or functions to the fire model input. The most important source term in the simulation is the rate of heat release (RHR). The RHR can be defined using analytical curves, like $t^{2}$-curve (Heskestad and Delichatsios, 1977; NFPA, 1985), or specific experimental curves.

Typical results of the fire simulation are gas temperatures, smoke layer position and temperature of some solid object like cable. Usually, the actual target function is the time when some event takes place. Some examples of the target functions are smoke filling time, flash over time and component failure time. In this work, the most important target function is the cable failure time. An analytical, time dependent solution of the axially symmetric heat transfer equation is used to calculate the cable core and surface temperatures. The boundary condition is the gas temperature, given by CFAST. The analytical solution is relatively easy to implement, but the actual calculation may be time consuming, because the solution involves the roots of a non-linear equation and long convolution sums. These operations are made faster by pre-computing and tabulating the roots in advance, and by re-arranging the convolution sums to a form, where only current, and previous time step values are needed. The accuracy of the model has been validated by comparing against 1-D finite element solutions of the same problem. The failure of the cable is assumed, when it reaches some predefined failure temperature, which may be chosen randomly to consider the uncertainty of the method.

\section{Results and discussion}

\subsection{Cable tunnel fire scenario}

A fire in the cable tunnel of a nuclear power plant is simulated to find out the failure probability of cables located in the same tunnel with the fire. The fire ignites in a cable tray and the fire gases heat up a redundant cable, located on the opposite side of the same tunnel. The effect of a screen that divides the tunnel between the source and target is also studied. The distribution of the heat detector activation times is also calculated. A plan view of the physical geometry and the corresponding CFAST model are outlined in Fig. 1. A vertical cut of the tunnel is shown in Fig. 2. The tunnel is divided into five virtual rooms to allow horizontal variations in layer properties. The fire source is located in ROOM 1 and the target cable in ROOM 3, just on the opposite side of the screen. The length of the screen does not cover the whole tunnel, and therefore it is possible that smoke flows around the screen to the target. It is also possible, that smoke flows below the screen. In the end of the tunnel is a door to the ambient. The RHR from the fire source is modelled using an analytical $t^{2}$-type curve

$\dot{Q}(t)(\mathrm{kW})=\min \left\{\dot{Q}_{\max }, 1000\left(\frac{t}{t_{\mathrm{g}}}\right)^{2}\right\}$

where $t$ is time, $t_{\mathrm{g}}$ is the RHR growth time and $\dot{Q}_{\text {max }}$ is the maximum RHR, that depends on the tunnel size. 


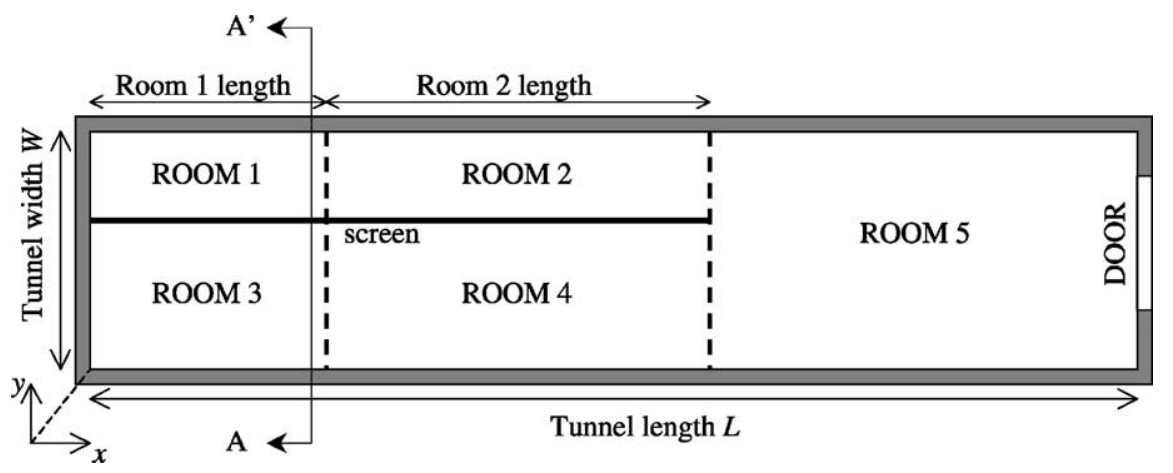

Fig. 1. A plan view of the cable tunnel model.

$t_{\mathrm{g}}$ is treated as a normally distributed random variable with mean of $1000 \mathrm{~s}$ and standard deviation of $300 \mathrm{~s}$.

Two different versions of the screen are studied. First, the screen is assumed to exist, with the lower edge below the $50 \%$ of the tunnel height. In the second scenario, the screen is removed by setting the lower edge very close to the ceiling, retaining the virtual room structure of the model. The dimensions of the tunnel and the cable tray locations are taken from the measured distributions of the power plant. About 50 tunnel cross sections have been studied to generate the distributions. The distribution of the cable diameter is based on the measurements in seven tunnel cross sections, containing 815 cables. A complete list of the random variables is given in Table 1. Fictitious, but typical values are assumed for the heat detector properties. Detector Response Time Index (RTI) determines the thermal inertia of the detector. While most of the variables are true physical properties and dimensions, the lengths of the virtual rooms are purely associated to

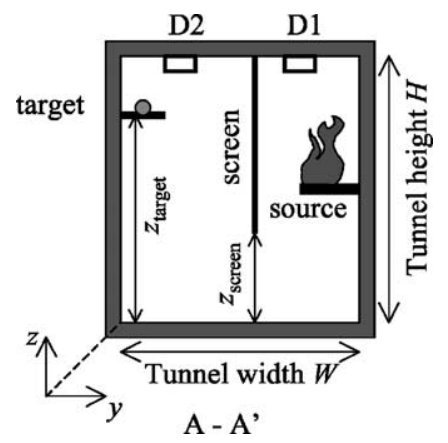

Fig. 2. A vertical cut $\mathrm{A}-\mathrm{A}^{\prime}$ of the cable tunnel model. D1 and D2 denote heat detectors 1 and 2, respectively. the numerical model. It is desirable that these variables are less important than the true physical variables.

Three output variables are considered:

1. Failure time of the target cable.

2. Activation time of the first heat detector (D1), located in the room of fire origin.

3. Activation time of the second heat detector (D2), located in the room of target cable.

The Monte Carlo simulations were performed for both scenarios to generate the distributions of the output variables, and to find out the importance of each input variable. The convergence of the simulations was ensured by monitoring the values of the $10,20, \ldots, 90 \%$ fractiles, mean values and standard deviations of the output variable distributions. The convergence was assumed, when the values changed less than $1.5 \%$ in 50 iterations. About 1000 iterations were needed to reach the convergence. The simulations took typically about 1 day on a $1.7 \mathrm{GHz}$ Pentium Xeon processor.

The distributions of the target failure times are shown in Fig. 3. The overall failure probabilities cannot be derived from the simulations because the distributions do not reach their final values during the total simulation time of $2400 \mathrm{~s}$. However, the existence of the screen is found to dramatically decrease the failure probability of the redundant cable.

The distributions of the activation times of fire detectors D1 and D2 are shown in Figs. 4 and 5, respectively. The activation time distributions of detector D1 are very narrow. For D2, the distributions are wider than for D1, and the existence of the screen decreases the activation probability from 1.0 to 0.8 . 
Table 1

A list of random variables used in the cable tunnel fire scenario

\begin{tabular}{|c|c|c|c|c|c|c|}
\hline Variable & Distribution & Mean & S.D. & Min & Max & Units \\
\hline RHR growth time $t_{\mathrm{g}}$ & Normal & 1000 & 300 & 0 & 3000 & $\mathrm{~s}$ \\
\hline Source height $z_{\text {source }} / H$ & Uniform & & & 0 & 0.7 & \\
\hline Ambient temperature & Normal & 20 & 3.0 & & & ${ }^{\circ} \mathrm{C}$ \\
\hline Tunnel height $H$ & Measured & & & 1 & 8 & $\mathrm{~m}$ \\
\hline Tunnel width $W_{\text {tunnel }}$ & Measured & & & 1.5 & 6.5 & $\mathrm{~m}$ \\
\hline ROOM 1 length & Uniform & & & 2 & 5 & $\mathrm{~m}$ \\
\hline ROOM 2 length & Uniform & & & 5 & 10 & $\mathrm{~m}$ \\
\hline Tunnel length $L$ & Uniform & & & 30 & 100 & $\mathrm{~m}$ \\
\hline Door height $z_{\text {door }} / H$ & Uniform & & & 0.1 & 1.0 & \\
\hline Door width $W_{\text {door }} / W_{\text {tunnel }}$ & Uniform & & & 0.01 & 1.0 & \\
\hline Screen edge height $z_{\text {screen }} / H$ & Uniform & & & $0 / 0.95$ & $0.5 / 1.0$ & \\
\hline Dimensionless cable height $z_{\text {target }} / H$ & Measured & & & 0.34 & 1 & \\
\hline Cable radius & Measured & & & 9 & 81 & $\mathrm{~mm}$ \\
\hline Critical cable temperature & Normal & 200 & 20 & & & ${ }^{\circ} \mathrm{C}$ \\
\hline Cable conductivity & Normal & 0.16 & 0.05 & 0.1 & 0.5 & $\mathrm{~W} / \mathrm{km}$ \\
\hline Cable density & Normal & 1400 & 200 & 1000 & 2000 & $\mathrm{~kg} / \mathrm{m}^{3}$ \\
\hline Detector activation temperature & Normal & 57 & 3 & & & ${ }^{\circ} \mathrm{C}$ \\
\hline Detector RTI & Normal & 50 & 10 & 40 & 60 & $(\mathrm{~m} / \mathrm{s})^{1 / 2}$ \\
\hline Ventilation time constant & Uniform & & & 0.5 & 10 & $\mathrm{~h}$ \\
\hline Concrete density & Uniform & & & 1500 & 3000 & $\mathrm{~kg} / \mathrm{m}^{3}$ \\
\hline
\end{tabular}

The probability that the fire is detected before the target failure is studied in Fig. 6 by plotting the failure times of both scenarios against the corresponding detection times. In all cases, the detection takes place before the failure. However, this does not tell about the probability of the fire extinction because the sprinkler reliability and suppression processes are not considered.

The sensitivity of the target failure time for the various input variables is studied by calculating the

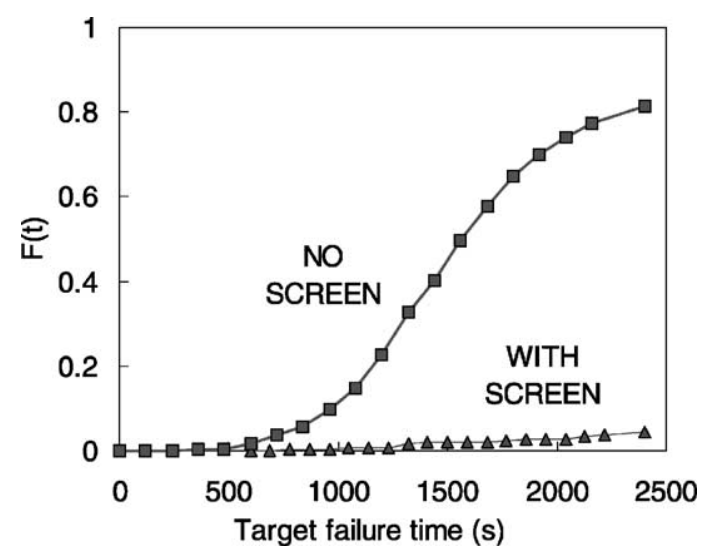

Fig. 3. The distributions of the target cable failure time. rank order correlations. A graphical presentation of the rank order correlation coefficients is shown in Fig. 7. For clarity, only the values corresponding to the situation without a screen are shown. The three most important variables are the RHR growth time, assumed cable failure temperature and the cable radius. As the distributions of the first two variables do not have a solid physical background, this result can be used to direct the future research. In addition, the length of the virtual fire room has a strong correlation

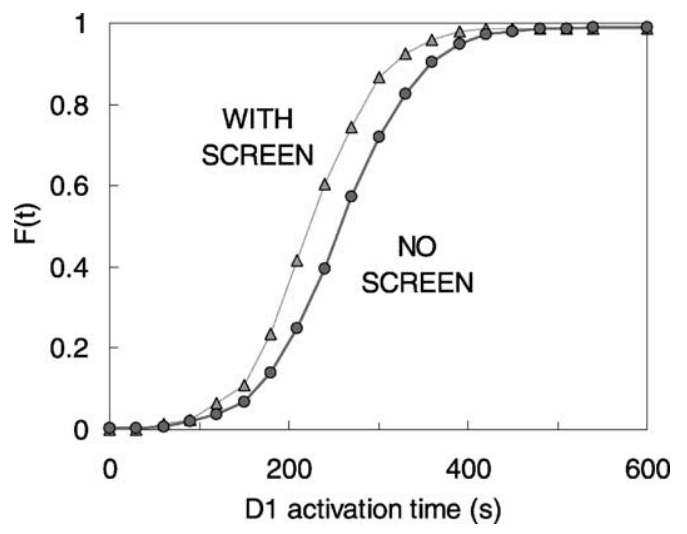

Fig. 4. The distributions of the heat detector D1 activation times. D1 is located inside the fire room (ROOM 1). 


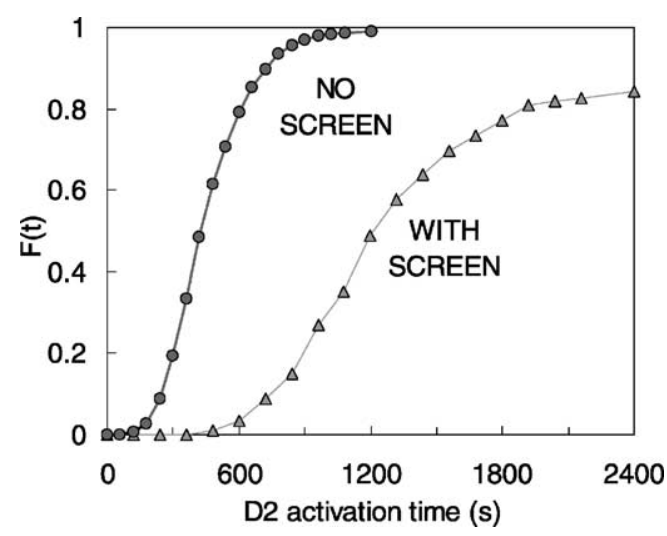

Fig. 5. The distributions of the heat detector D2 activation times. D2 is located inside the target room (ROOM 3).

with the failure time. In the scenario where screen is present, the height of the lower edge of the screen has strong negative correlation with the failure time. It is therefore recommended, that the screen, when present, should reach as low as possible to prevent smoke from flowing under the screen. The correlations with absolute value less than about 0.2 are not significant.

\subsection{Electronics room fire scenario}

As a second example, a fire of an electronics cabinet inside the electronics room is studied. In their previous study of the similar fire, Eerikäinen and Huhtanen (1991) found that a fire of a single cabinet does not cause direct threat to the other cabinets, in terms of the

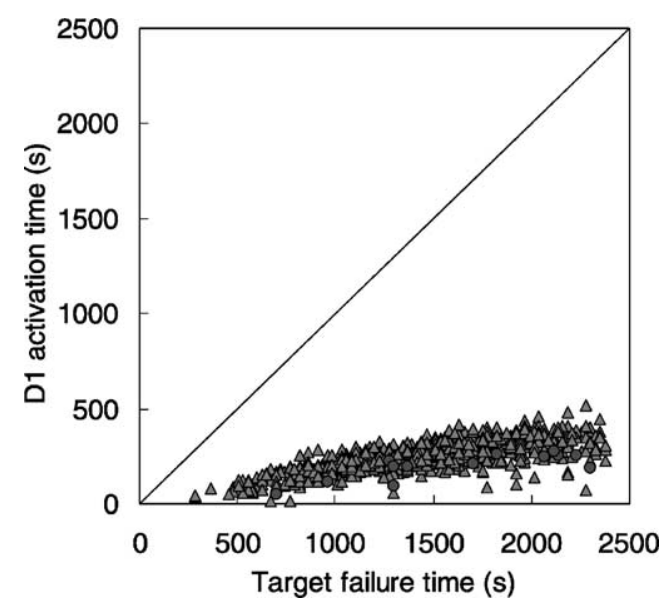

Fig. 6. Comparison of D1 activation times vs. target failure times $(N=954)$.

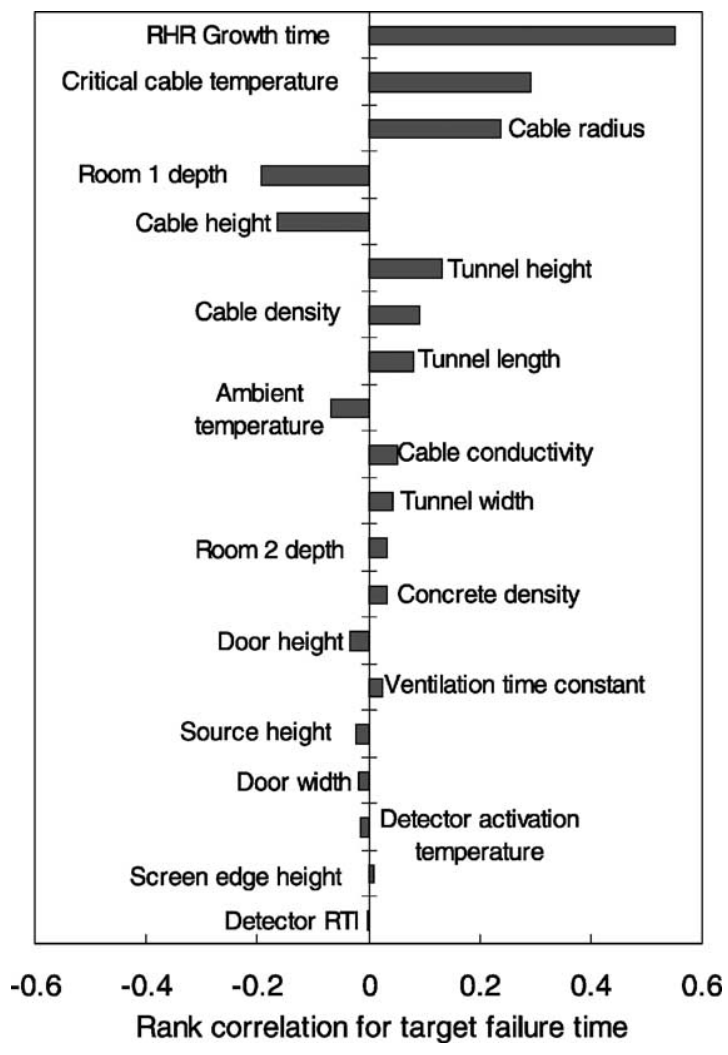

Fig. 7. The sensitivity of the target failure time to the input variables in the cable tunnel scenario.

gas temperature. However, the spreading of the fire, conductive heating of the components in the neighbour cabinets and the effect of smoke on the electronic components may cause failures in the other cabinets of the fire room. Therefore, the probability that the room is filled with smoke during a fire must be considered in addition to the temperature increase.

The fire room is $18.5 \mathrm{~m}$ long, $12.1 \mathrm{~m}$ wide and $3.0 \mathrm{~m}$ high electronics room, containing about 100 electronics cabinets in 12 rows. The geometry is outlined in Fig. 8. The room has mechanical ventilation with a nominal flow rate $\left(1.11 \mathrm{~m}^{3} / \mathrm{s}\right)$ and some additional leakages to the ambient. Both the ventilation flow rate and the leakage area are taken to be random variables. Separate cooling devices and smoke circulation through the ventilation system are not taken into account, nor is the heat transfer to the room boundaries. The omission of the cooling devices is known to change the predicted gas temperature sig- 


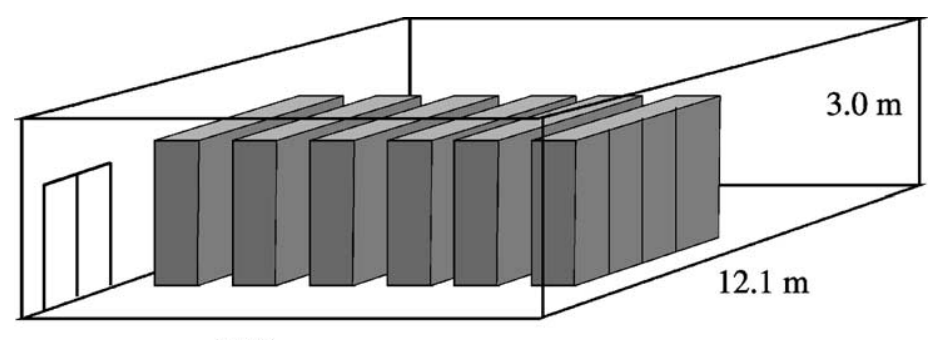

$18.5 \mathrm{~m}$

Fig. 8. An outline of the electronics room fire scenario.

nificantly. The results should therefore be considered as indicative only.

The starting point of the simulation is the collection of the physical data on the electronic cabinets. The geometrical properties and fuel content of 98 cabinets were measured. Based on the collected information, the cabinets were grouped into seven groups, shown in Table 2. The second column shows the number of cabinets in each group. $P_{\text {ign }}$ is the probability, that the ignition takes place in the particular group, calculated based on the amount of electronic devices (circuit boards and relays) inside the cabinets. Most differences are found in the fuel surface area $A_{\text {fuel }}$ and fire load. As these properties have an effect on the heat release rate curve, they are chosen to be distinctive properties like the vent properties.

The RHR is calculated using a cabinet model of Keski-Rahkonen and Mangs (2003), that assumes that the heat release rate during the fully developed cabinet fire is determined by the ventilation openings in the upper and lower parts of the cabinet. The maximum RHR is given by

$\dot{Q}_{\text {max,vent }}(\mathrm{kW})=7400 \chi \sqrt{\frac{H_{\mathrm{v}}}{\left(2.3 / A_{\mathrm{e}}^{2}\right)+\left(1 / A_{\mathrm{i}}^{2}\right)}}$ where $\chi$ is combustion efficiency factor, $H_{\mathrm{v}}$ is the height between the openings and $A_{\mathrm{e}}$ and $A_{\mathrm{i}}$ are the areas of the exhaust and inflow openings, respectively. During the fire, the cabinet doors may open due to the thermal effects. In this case, the heat release rate is not ventilation but fuel controlled, and the maximum RHR becomes

$\dot{Q}_{\text {max }, \text { fuel }}=\dot{Q}_{\text {fuel }}^{\prime \prime} \times A_{\text {fuel }}$

where $\dot{Q}_{\text {fuel }}^{\prime \prime}$ is the nominal heat release rate per unit surface of fuel, a random variable in the simulation, and $A_{\text {fuel }}$ is the free fuel area. A door opening indicator is used to select which equation is used to calculate the maximum RHR. The probability of the door-opening event during the fire is assumed to be 0.50 . A $t^{2}$-type RHR curve with an exponential decay rate is used.

$$
\begin{aligned}
\dot{Q}(t)(\mathrm{kW})= & \min \left\{1000\left(\frac{t}{t_{\mathrm{g}}}\right)^{2}, \dot{Q}_{\max },\right. \\
& \left.\dot{Q}_{\max } \exp \left(-\frac{t-t_{\mathrm{d}}}{\tau}\right)\right\}
\end{aligned}
$$

where $\dot{Q}_{\max }$ is either $\dot{Q}_{\text {max, vent }}$ or $\dot{Q}_{\text {max,fuel }}$, depending on the value of the door opening indicator. The

Table 2

\begin{tabular}{|c|c|c|c|c|c|c|c|c|c|}
\hline Cabinet group & $\begin{array}{l}\text { Number of } \\
\text { cabinets }\end{array}$ & $P_{\text {ign }}$ & $H_{\text {cabinet }}(\mathrm{m})$ & $H_{\mathrm{v}}(\mathrm{m})$ & $A_{\mathrm{e}}\left(\mathrm{m}^{2}\right)$ & $A_{\mathrm{i}}\left(\mathrm{m}^{2}\right)$ & $\dot{Q}_{\max , \text { vent }}(\mathrm{kW})$ & $A_{\text {fuel }}\left(\mathrm{m}^{2}\right)$ & Fire load (MJ) \\
\hline HA & 42 & 0.53 & 2.33 & 1.96 & 0.126 & 0.121 & 425.8 & 5.35 & 1520 \\
\hline $2 \mathrm{HA}$ & 9 & 0.18 & 2.36 & 1.97 & 0.129 & 0.110 & 419.5 & 8.54 & 2700 \\
\hline $2 \mathrm{HD}$ & 1 & 0.02 & 2.36 & 1.97 & 0.129 & 0.110 & 419.5 & 7.76 & 2000 \\
\hline JB & 14 & 0.08 & 2.36 & 1.97 & 0.129 & 0.110 & 419.5 & 2.49 & 800 \\
\hline JK2 & 3 & 0.05 & 2.20 & 2.05 & 0.015 & 0.068 & 63.8 & 7.37 & 1200 \\
\hline JM & 17 & 0.13 & 2.36 & 1.97 & 0.129 & 0.110 & 419.5 & 3.22 & 800 \\
\hline $\mathrm{P}$ & 5 & 0.01 & 2.19 & 1.89 & 0.215 & 0.126 & 574.4 & 0.64 & 100 \\
\hline
\end{tabular}

Grouping of the electronic cabinets

The symbols are explained in the text. 
Table 3

A list of random variables used in the electronics room fire scenario

\begin{tabular}{|c|c|c|c|c|c|c|}
\hline & Distribution type & Mean & S.D. & Min & Max & Units \\
\hline RHR growth time $t_{\mathrm{g}}$ & Uniform & & & 750 & 2000 & $\mathrm{~s}$ \\
\hline Door opening indicator & Discrete & & & 0 & 1 & \\
\hline RHR decay time $\tau$ & Uniform & 800 & 1400 & 800 & 1400 & $\mathrm{~s}$ \\
\hline Cabinet type & Measured & & & 1 & 7 & \\
\hline RHR per unit area $\dot{Q}_{\text {fuel }}^{\prime \prime}$ & Normal & 150 & 40 & 50 & 300 & $\mathrm{~kW} / \mathrm{m}^{2}$ \\
\hline Detector RTI & Normal & 80 & 10 & 50 & 100 & $(\mathrm{~m} / \mathrm{s})^{1 / 2}$ \\
\hline Detector activation temperature & Normal & 57 & 5 & 40 & 100 & ${ }^{\circ} \mathrm{C}$ \\
\hline Critical target temperature & Normal & 80 & 10 & 50 & 200 & ${ }^{\circ} \mathrm{C}$ \\
\hline Target radius & Uniform & & & 1 & 5 & $\mathrm{~mm}$ \\
\hline Leakage area & Uniform & & & 0 & 3 & $\mathrm{~m}^{2}$ \\
\hline Virtual source height $z_{f} / H_{\text {cabinet }}$ & Uniform & & & 0 & 0.5 & \\
\hline Ventilation flow rate & Normal & 1.11 & 0.2 & 0.5 & 2 & $\mathrm{~m}^{3} / \mathrm{s}$ \\
\hline
\end{tabular}

growth and decay time constants, $t_{\mathrm{g}}$ and $\tau$, are random variables in the simulation. The parameters of their distributions are taken from the experimental works of Mangs and Keski-Rahkonen (1994, 1996). The decay phase starts $\left(t=t_{\mathrm{d}}\right)$ when $70 \%$ of the fire load is used.

It is very difficult to apply the zone-type fire model to the cabinet fire, because the actual source term for the room is the smoke plume flowing out of the cabinet. A reasonable representation of the smoke flow can be found if the base of the "virtual" fire source is placed close to one-half of the cabinet height. Then the mass flow of free smoke plume at the height of the cabinet ceiling is roughly equal to the vent flow. Now, the height of the virtual fire source is considered as a random variable. A list of the random variables is given in Table 3.

Four output variables are considered

1. Failure time of the target cable. The cable is located $0.5 \mathrm{~m}$ below the height of the cabinet, therefore simulating a device inside a similar cabinet.

2. Smoke filling time: time when the smoke layer reaches the height $2.0 \mathrm{~m}$.

3. Smoke filling time: time when the smoke layer reaches the height $1.5 \mathrm{~m}$.

4. Smoke filling time: time when the smoke layer reaches the height $1.0 \mathrm{~m}$.

The effect of the observation height is studied by observing the smoke filling at three different heights. The convergence was ensured in the same manner, as in the previous example. Seven hundred iterations were needed for convergence this time. The simulation took about $4 \mathrm{~h}$ on a $2.0 \mathrm{GHz}$ Xeon processor.

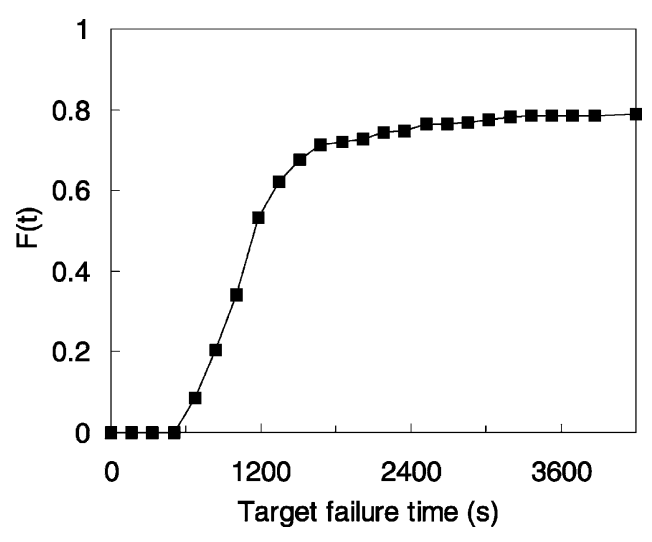

Fig. 9. The distribution of the target failure time.

The distribution of the target failure times is shown in Fig. 9. Target failures start after $500 \mathrm{~s}$, after which the failure probability increases to 0.8 . Higher failure probability would be found inside the neighbouring cabinets with a direct contact to the burning cabinet, and in the ceiling jet of the fire gases. These results are inconsistent with the previous findings of Eerikäinen and Huhtanen (1991), who concluded, based on the CFD simulations, that the mean gas temperatures in the room do not become high enough for component failures. The most obvious reason for the inconsistency is the difference in the simulation times. The overall simulated period employed here was $7200 \mathrm{~s}$, while Eerikäinen and Huhtanen stopped their simulation after $600 \mathrm{~s}$. At this point, our failure probability is still very small. Other possible reasons for the inconsistency are the omission of the cooling devices 


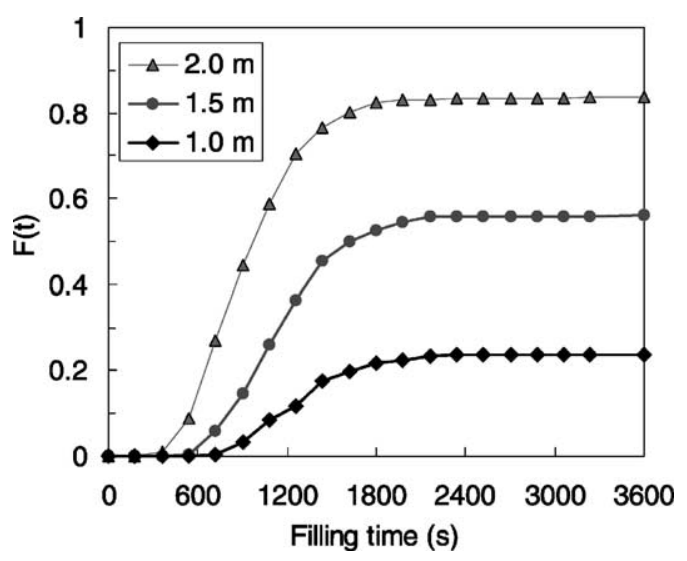

Fig. 10. The distributions of the smoke filling time.

and the variation of the RHR curve, which may cause very severe conditions in some simulations.

The distributions of the smoke filling times are shown in Fig. 10. The smoke layer reaches the height of the electronic cabinets from 500 to $1800 \mathrm{~s}$ after the ignition. The overall probability of getting inside the smoke layer drops from 0.8 to 0.2 , when the observation height is reduced from 2.0 to $1.0 \mathrm{~m}$.

The sensitivity of the failure time to the input variables is shown in Fig. 11. The height of the virtual fire source has the strongest effect on the failure time. This is very unfortunate, because in this particular

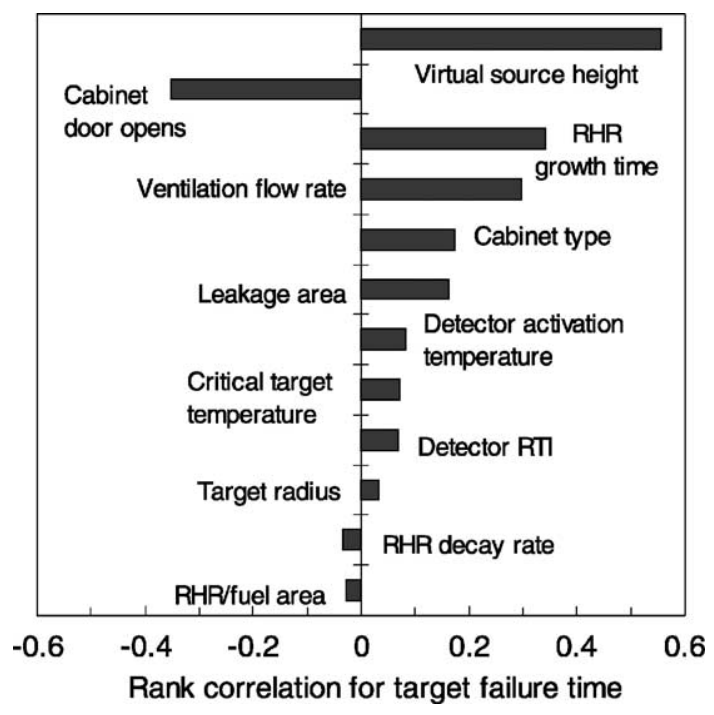

Fig. 11. The sensitivity of the target failure time to the input variables in the electronics room scenario. application it is a numerical parameter. Other important variables are the door opening indicator, RHR growth time and the ventilation flow rate. The reliable locking of the cabinet doors is the most important physical variable that can be directly affected by engineering decisions.

\section{Summary}

Probabilistic Fire Simulator is a tool for Monte Carlo simulations of fire scenarios. The tool is implemented as a worksheet computing tool, using commercial @RISK package for Monte Carlo part. The fire is modelled using two-zone model CFAST. The Monte Carlo simulations can provide the distributions of the output variables and their sensitivities to the input variables. Typical outputs are for example the times of component failure, fire detection and flashover. The tool can also be used as a worksheet interface to CFAST. Extension to the other fire models is possible.

The presented example cases demonstrate the use of the tool. Best available information on the input distributions was used in the cable tunnel scenario, which can therefore be considered as a realistic representation of the problem. The results of the tunnel scenario show that the heat detector gives an alarm before the loss of the redundant cables, with a very high probability. However, the detector reliability was not considered. According to the sensitivity measures, the most important parameters for the safety of the redundant cables are (i) the growth rate of the fire, (ii) the screen providing a physical separation of the burning and target cable trays, (iii) the critical temperature of the cable material and (iv) the radius (mass) of the cable. Unfortunately, the failure time is also sensitive to the length of the virtual room of fire origin, which is a purely numerical parameter. This phenomenon should be studied more carefully in the future.

The second example considered an electronic cabinet fire inside an electronics room. The procedure of data collection, model development and actual simulation were described to demonstrate the use of the model in practical applications. The data collection and analysis was found to be the most time consuming part of the process. The various ways to enhance this kind of analysis are therefore needed. The simulation results showed that during a fire of a single cabinet, 
the thermal environment inside the room might cause a failure of an electronic component, with a probability of 0.8 . The inconsistency with the earlier studies can be explained with the longer simulation time and the variation of the RHR curve. Due to the strong effect of opening the cabinet door on the RHR curve, a reliable locking of the cabinet doors is the most important physical variable that can be directly affected by engineering decisions.

\section{Acknowledgements}

The contribution of Dr. Timo Korhonen from VTT during the data collection is greatly appreciated. This study has been financed by the Finnish Centre for Radiation and Nuclear Safety, the Ministry of Trade and Industry, IVO Power Engineering Ltd. (now Fortum) and TeollisuudenVoima $\mathrm{Oy}$.

\section{References}

Eerikäinen, L., Huhtanen, R., 1991. Ydinvoimalaitoksen huonetilapalo, Loviisa 1:n valvomorakennuksen huonetilan palon numeerinen tarkastelu (Room fire in a Nuclear Power Plant, Numerical Study of the Room Fire in a Control Building of Loviisa 1 Power Plant). Finnish Centre for Radiation and Nuclear Safety, Helsinki. Report STUK-YTO-TR 30, 59 pp. + App. 26 pp.

Hofer, E., 1999. Sensitivity analysis in the context of uncertainty analysis for computationally intensive models. Comp. Phys. Commun. 117, 21-34.

Heskestad, G., Delichatsios, M.A., 1977. Environments of Fire Detectors. Phase 1. Effect of Fire Size, Ceiling Height and Materials. Vol. 2. Analysis. NBS GCR 77-95, National Bureau of Standards. Gaithersburg, MD, 129 pp.
Keski-Rahkonen, O., Hostikka, S., 1999. Theory versus experiment-did you forget design of experiment and data reduction? Structural Mechanics in Reactor Technology 15, Post Conference Seminar No. 6, Fire Safety in Nuclear Power Plants and Installations. Munich-Garching, Germany, September 6-7, $21 \mathrm{pp}$.

Keski-Rahkonen, O., Mangs, J., 2003. Quantitative determination of source terms from fires in electronic cabinets: maximum rate of heat release, minimum rate of heat release needed for flashover, and effluent yields from incomplete combustion. Fire Safety J., submitted for publication

Mangs, J., Keski-Rahkonen, O., 1994. Full scale fire experiments on electronic cabinets. Espoo, Technical Research Centre of Finland. VTT Publications 186, 50 pp. + App. 37 pp.

Mangs, J., Keski-Rahkonen, O., 1996. Full scale fire experiments on electronic cabinets II. Espoo, Technical Research Centre of Finland. VTT Publications 269, 48 pp. + App. 6 pp.

Mangs, J., Keski-Rahkonen, O., 1997. Full-scale fire experiments on vertical and horizontal cable trays. Technical Research Centre of Finland, Espoo. VTT Publications 324, 58 pp. + App. 44 pp.

McKay, M.D., Beckman, R.J., Conover, W.J., 1979. A comparison of three methods for selecting values of input variables in the analysis of output from a computer code. Technometrics 21 (2), 239-245.

NFPA, 1985. NFPA 204M, Guide for Smoke and Heat Venting, National Fire Protection Association, Quincy, MA.

Peacock, R.D., Forney, G.P., Reneke, P., Portier, R., Jones, W.W., 1993. CFAST, the Consolidated Model of Fire Growth and Smoke Transport. Gaithersburg, National Institute of Standards and Technology, NIST Technical Note 1299. 118 pp. + App. 116 pp.

Pebesma, E.J., Heuvelink, G.B.M., 1999. Latin hypercube sampling of multigaussian random fields. Technometrics 41 (4), 303-312.

Spiegel, M.R., 1980. Theory and Problems of Probability and Statistics, SI ed. McGraw-Hill, New York, 372 pp.

Stein, M.L., 1987. Large sample properties of simulations using latin hypercube sampling. Technometrics 29 (2), 143-151.

Vose, D., 1996. Quantitative risk analysis: a guide to Monte Carlo simulation modelling. Wiley, Chichester, England, 328 pp. 